\title{
Cytokine Varieties and Cytokine Storms in COVID-19: A Review
}

\author{
A. S. M. Giasuddin ${ }^{1, *}$, K. A. Jhuma ${ }^{1}$, R. S. Giasuddin ${ }^{2}$, W. A. Chowdhury ${ }^{1}$ \\ ${ }^{1}$ Impulse Hospital, ImHS\&RCLtd, 304/E Tejgaon Industrial Area, Dhaka-1208, Bangladesh. \\ ${ }^{2}$ Department of Medicine, Anwer Khan Modern Medical College, Road No. 8A, Dhanmondi, Dhaka-1205, Bangladesh.
}

How to cite this paper: A. S. M. Giasuddin, K. A. Jhuma, R. S. Giasuddin, W. A. Chowdhury. (2022) Cytokine Varieties and Cytokine Storms in COVID-19: A Review. International Journal of Clinical and Experimental Medicine Research, 6(1), 16-23. DOI: 10.26855/ijcemr.2022.01.004

Received: October 9, 2021

Accepted: October 31, 2021

Published: November 29, 2021

*Corresponding author: A. S. M. Giasuddin, Impulse Hospital, ImHS\&RCLtd, 304/E Tejgaon Industrial Area, Dhaka-1208, Bangladesh. Email: asmgias@hotmail.com

\begin{abstract}
Cytokines are heterogeneous group of protein cell regulators having many (pleotropic) activities produced by a wide variety of cells in the body. They have made tremendous impact in clinical medicine as well as laboratory medicine. Therapeutic impacts of cytokines have been felt in the areas of cancer, infectious diseases, blood disorders, rheumatic and autoimmune diseases mainly. Quantitative determination of the levels of various cytokines in blood and other body fluids were found to be useful in order to monitor disease activity or monitor treatment or evaluate the need for treatment. The infectious disease caused by severe acute respiratory syndrome coronavirus-2 (SARS-CoV-2) is termed as Corona Virus Disease 2019 (COVID-19). This new virus and the disease were unknown before the outbreak in Wuhan, China in December 2019. Clinical presentations of COVID-19 are a vast spectrum ranging from asymptomatic carriers to critically ill patients with multi organ failure needing multiple life supports. After the virus gains entry into the body through the mucus membrane, massive amounts of cytokines production i.e. cytokine storms occur which play a pivotal role behind COVID-19 pathophysiology in severe and critically ill patients. The cytokines involved are IL-1, IL-2, IL-6, IL-7, IL-8, IL-10, IL-17, IFN- $\alpha$, IFN- $\gamma$, G-CSF, GM-CSF, TNF- $\alpha$, TNF- $\beta$, MCP-1, MIP1A, etc. Potentially dangerous complications may occur which demand that highly trained and well-informed clinicians, nursing staff and laboratory medicine manpower are available where cytokines are to be introduced either as therapy or clinical trial. Some recent aspects of these cytokine varieties and cytokine storms in COVID-19 were discussed in this review article.
\end{abstract}

\section{Keywords}

Cytokine, SARS-CoV-2, COVID-19, Clinical Medicine, Laboratory Medicine

\section{What are Cytokines?}

The cytokines are heterogeneous group of protein cell regulators, variously called lymphokines, monokines, interleukins and interferons. They are produced by a wide variety of cells in the body, play an important role in the pathophysiology of range of diseases and have therapeutic potential. These heterogenous group of proteins have a number of characteristics in common: (i) cytokines are low molecular weight $(<80 \mathrm{KDa})$ secreted proteins which are often glycosylated; (ii) they are usually produced transiently and locally, acting in a paracrine or autocrine, rather than endocrine, manner; (iii) they are involved in immunity and inflammation where they regulate the ampli- 
tude and duration of response; (iv) they are extremely potent, generally acting at picomolar concentrations; (v) they interact with high affinity cell surface receptors specific for each cytokine or cytokine group; (vi) their cell surface binding ultimately leads to a change in the pattern of cellular RNA and protein synthesis, and to altered cell behavior; (vii) individual cytokines have multiple overlapping cell regulatory actions; (viii) cytokines interact in a network or a series of cascades by: first, inducing each other; second, trans modulating cytokine cell surface receptors and third, by synergistic, additive or antagonistic, interactions on cell function. The response of a cell to a given cytokine is dependent on the local concentration of the cytokine, the cell type and other cell regulators to which it is concomitantly exposed. Thus, the cytokines are usually secreted as a result of new synthesis by the producer cell in response to inducing stimuli. In most cases, signals are sent to the nucleus of the target cells causing changes in gene expression [1-3]. The fundamental elements of the three types of cytokine-cell interaction are autocrine, paracrine and endocrine types. Autocrine stimulation occurs when a cell possesses receptors to respond to cytokines it produces itself; paracrine type of cytokine-cell interaction is a localized stimulation that can occur if a different cell type, near the producer cell, has the appropriate receptors; endocrine type of cytokine-cell interaction means cytokines may be released in the blood stream or other body fluids that interact with cells elsewhere in the body. Identification and characterization of the many cytokines now known has resulted in a variety of names and classifications. However, there is considerable overlap in the biological effects and mechanisms of cytokines actions currently placed in different categories. It is important to mention that the name given to a cytokine is often not a very useful guide to its biological functions, e.g. tumor necrosis factor is of interest more for its role in inflammation rather than tumor killing [2-11].

\section{Cytokines with So Many (Pleotropic) Activities: Why?}

In an elegant review, Balkwill and Burke tried to explain the interactions of cytokines among various cell types, although it is difficult to understand the complexity of the cytokine network from our current knowledge. In an attempt to answer the questions posed, the following reasons may be proposed: (i) the complexity of concurrent messages that any one cell can receive may result in a multiplicity of checks and balances that limit the duration and extent of a cellular response; (ii) another explanation for the complexity of cytokine number and function is that different induction signals result in the production of distinct cytokines with a similar range of actions; (iii) in addition, different subsets of a cell types may produce a distinct spectrum of cytokines. For example, TH1 subset of human CD+ T-lymphocytes produces pre-dominantly IL-2 and IFN- $\gamma$, but not IL-4 and IL-5 whereas TH2 subset of $\mathrm{CD}_{4}+$ T-lymphocytes synthesize and secret IL-4 and IL-5 but not IL-2 and IFN- $\gamma$. Such selective production may be one of the mechanisms for controlling the potentially pleiotropic effects of cytokines to their appropriate responder cells. Otherwise, given the net balance required to maintain healthy tissues, it is not surprising that abnormal cytokine activity may be seen in many diseases [2-8].

\section{Impact of Cytokines in Clinical Medicine}

The therapeutic impact of cytokines has been felt mainly in the fields of infectious diseases, cancer, blood disorders, rheumatic diseases and autoimmune diseases as briefly noted below [2-11].

(i) Infectious Diseases: The most likely used cytokine has been IFN- $\alpha$, which has US-FDA approval for chronic hepatitis B and for hepatitis C. It has also shown promise in other viral conditions, including laryngeal papilloma, warts, common cold (prophylactically only) and, though controversial, AIDS. IFN- $\gamma$ has been shown to be of striking benefit in chronic granulomatous disease in cutting down the incidence of serious infections. In the vaccine front, there is evidence that IFN- $\gamma$, IL-1 and IL-2 may have useful adjuvant activity, particularly in immune-compromised patients. A clinical role for inhibitors of cytokines, particularly antibodies to the cytokines (e.g. anti-TNF, IL-10) has also been suggested in life-threatening conditions notably septic shock due to Gram negative infection and cerebral malaria.

(ii) Cancer: The majority of successes in clinical trials with cytokines against cancer have been obtained with IFN- $\alpha$ and IL-2. Currently, IFN- $\alpha$ has the approval for the treatment of Hairy Cell Leukemia, Kaposi's sarcoma and Basal Cell Carcinoma, and is considered effective in a variety of other tumors. Also, considerable effort is being given to IFN/drug combinations and combined therapy with IFN and surgery, radiation, hyperthermia, monoclonal antibodies, etc. IFN is usually given subcutaneously or intra-lesionally. Treatment with IFN tends to be long-term (months-years), which makes it very expensive. IFN- $\gamma$ has not shown consistent therapeutic potential in any malignancies. IL-2 is licensed in all European community countries for the treatment of metastatic renal cell carcinoma and licensing is expected for melanoma. Extensive trials with TNF- $\alpha$ have been disappointing and li- 
mited by toxicity, but it may be of value in treating ascites of ovarian carcinoma.

(iii) Blood Disorders: Chemotherapy for malignant diseases and intensive therapy used with bone-marrow transplantation often leads to acquired neutropaenia. Both GM-CSF and G-CSF have been shown to accelerate neutrophil recovery in post chemotherapy neutropaenia. Clinical trials in patients with lung cancer have shown that the G-CSF induced recovery of the post-chemotherapy neutropaenia is associated with fewer infections, fewer antibiotics, and a shorter period of stay in the hospital. Similar effects are observed with GM-CSF use after bone-marrow transplantation. G-CSF is now almost the standard therapy for the rare conditions of severe congenital neutropaenia. One of the remarkable features of G-CSF is its safety margin and almost complete absence of side-effects. Although GM-CSF shows similar efficacy to G-CSF in shortening chemotherapy-induced neutropaenia, it has some side effects which increase with modest increases in dose. G-CSF has FDA-US approval for use in post chemotherapy neutropaenia and GM-CSF has approval for use after bone-marrow transplantation.

(iv) Rheumatic and Autoimmune Diseases: The experimental evidence implicates cytokines in the pathogenesis of rheumatoid arthritis and osteoarthritis. These are two commonest causes of joint failure as cytokines are involved in the degeneration of connective tissue in cartilage and bone. The following therapeutic approaches with cytokines and antibodies to cytokines are already in the experimental stage of evaluation: replacement therapy with cytokine which might be deficient (e.g. IFN- $\gamma$, IL-4); neutralization of excessive amounts of cytokines (e.g. TNF, IL-1 $\alpha$, IL-1 $\beta$, IL-6, GM-CSF, IL-8) by monoclonal antibodies against these cytokines or their receptors; and administration of cytokines which are immunosuppressive or promote tissue healing (e.g. IL-10, TGF- $\beta$ ).

(v) Other Conditions: The renal conditions, IgA nephropathy and mesangial glomerulonephritis, have been shown experimentally to be benefited with anti-IL-6 therapy. There are some suggestions that anti-TNF might alleviate Graft-versus-Host disease. Anti-IL-4 therapy, might be useful in allergy by down-regulating IgE synthesis; while TGF- $\beta$ and IL-10 might be of value as a general cytokine inhibitor. A strong indication for cytokine therapy would be as replacement of a cytokine deficiency in production. While individual variations in the level of cytokines exist, no complete deficiency has yet been recognized.

Not surprisingly with such biologically potent molecules, toxicity (side effects) following cytokine therapy is common. For example, severe toxic effects of IFN $-\alpha$ include damage to liver, kidney, bone-marrow and heart. The treatment with IL-2 is complicated by vascular leak syndrome (hypotension, fluid retention and hepatic and renal failure together with nausea, vomiting and mental alterations-including) if treatment is continued. The occurrence of such potentially dangerous complications demands that highly trained and experienced physicians and nursing staffs must be available in hospitals where cytokines therapy is expected to be introduced either as therapy or as clinical trial.

\section{Impact of Cytokines in Laboratory Medicine}

With the availability of commercial cytokine assay kits, it seems to be useful to know the levels of various cytokines in blood and other body fluids in order to monitor disease activity or monitor treatment or evaluate the need for treatment. Although there is no situation at present where a cytokine assay can be said to be an essential part of management, perhaps the strongest case could be made for TNF levels in shock, since a raised level would be an indication for life-saving measure with anti-TNF. An excellent marker of flaring in inflammatory conditions is IL-6, being raised 24 hours before C-reactive protein. Levels of IL-1, TNF- $\alpha$, IL- 8 and TGF- $\beta$ in tissues and of IL-2 and TNF receptors in the joint fluids and blood, have also been shown to correlate with disease activity. Levels of TNF, IL-1 and IL-6 can also be predictive of inflammatory bowel disease and graft rejection well before it is clinically apparent. However, these commercially available cytokine assay kits are considered very expensive to be introduced in the routine diagnostic immunology laboratories. These assays should, therefore, be restricted to laboratories where professionally trained immunologist and technical staff are available, and where the results can be put ethically to further usage as research data, and where improvements in the efficacy and accuracy of assays are under constant surveillance [2-4, 9-11].

\section{What is COVID-19?}

COVID-19 is an infectious disease caused by the most recently discovered corona virus. Corona viruses are a large family of viruses which may cause illness in animals and humans. This new virus and the disease were unknown before the outbreak began in Wuhan, China in December 2019. The national health system and medical communities have encountered with unprecedented public health challenges at the beginning of 2020. A novel strain of corona virus infection has spread globally causing concern among public health experts. The causative 
agent for it has been finally identified to be 'severe acute respiratory syndrome coronavirus-2 (SARS-CoV-2) and termed the disease as corona virus disease of 2019 (COVID-19)'. Structurally, SARS-CoV-2 is an enveloped positive- sense RNA virus that belongs to the $\beta$-coronavirus genus with diameter about 65-125 nm containing a single strand of RNA coated by crown-like spikes on its outer surface as shown in Figure 1 [12-16].

SARS-CoV-2 has four main structural proteins including spike (S) glycoprotein, envelope (E) glycoprotein, membrane (M) glycoprotein, nucleocapsid $(\mathrm{N})$ protein, and also several non-structural proteins and multiple unique accessory proteins. The spike glycoprotein comprises two subunits which are responsible for the binding of the virus to the host cell receptor (S1 subunit) and the fusion of the virus to the cell membrane (S2 subunit). The nucleocapsid protein is located in the endoplasmic reticulum region and bound to nucleic acid material of the virus. This $\mathrm{N}$ protein is responsible for the viral genome and viral replication cycle. The membrane protein is the protein that gives structure to the virus and has a role in determining the shape of the virus envelope, whereas the envelop (E) protein has a role in the production and maturation of the virus as illustrated in Figure 1 [16-18].

The World Health Organization (WHO) raised the COVID-19 status from the public health emergency of international concern to a pandemic in March 2020. The disease has affected over 7 million people and claimed over 400,000 lives worldwide as of September 2020 [12, 14, 15, 18].

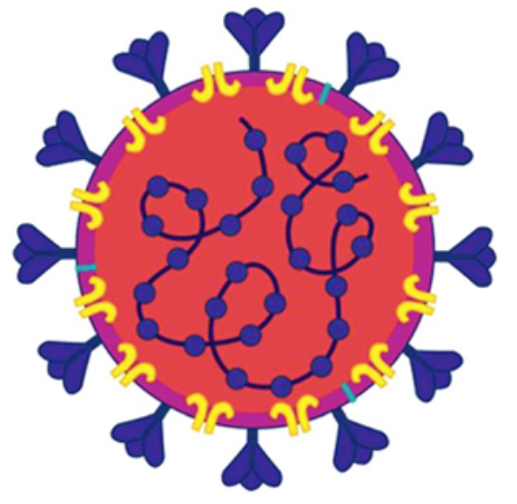

$\begin{array}{ll}\text { Species } & \text { SARS-CoV-2 } \\ \text { Family } & \text { Coronaviridae } \\ \text { Diameter } & 65-125 \mathrm{~nm}\end{array}$

Gene material ssRNA

Structural protein Spike (S) glycoprotein, envelope (E) glycoprotein, membrane $(\mathrm{M})$ glycoprotein, nucleocapsid $(\mathrm{N})$ protein

Characteristic findings Crown-like spikes (corona) on its outer surface.

Figure 1. The structure of SARS-CoV-19 [16, 17].

\section{Presenting Features and Mode of Spread of COVID-19}

The main presenting symptoms of COVID-19 are fever, headache, variable musculoskeletal involvement (myalgia specially), respiratory symptoms (cough, dyspnea, ARDS, etc), gastrointestinal and liver symptoms (diarrhea, abdominal pain, vomiting, nausea, etc), cutaneous symptoms e.g. skin rash with petechiae, etc. and laboratory findings such as thrombocytopenia, increased D-dimer, lymphopenia, leukopenia, etc. COVID-19 spread primarily from person to person through released droplets when someone sick sneezes or coughs which may land on the mouths or noses of people nearby. Also, close contract with someone sick-like hugging or shaking hands. The virus containing materials can be left on objects and surfaces such as furniture, flowers, utensils, books, roadside (footpath) materials, etc. If something contaminated are touched and then one touches face or another's face, one may get infected and might fall ill [18-20].

\section{Cytokine Storms in COVID-19}

Clinical presentations of COVID-19 are a vast spectrum ranging from an asymptomatic carrier to critically ill patients with multi organ failure needing multiple life supports. Regarding pathophysiology, the infection of SARS-CoV-2 is primarily from respiratory droplets through person to person transmissions and viral entry mainly through mucus membranes via nose, eyes and mouth [18-21]. After the virus gains entry through the mucous membrane, it is then directed into the pulmonary tissue, the type 2 pneumocyte in particular, via the angiotensin-converting enzyme 2 (ACE2). Direct pneumocyte infection and the viral cytopathic effect stimulate the innate immune system, which consists of monocytes, macrophage, and toll-like receptors, to produce various inflammatory cytokines and to stimulate the adaptive immune system. Subsequently, the activated adaptive immune system is liable for markedly increased inflammatory cytokines concentration [21, 22].

Many proposed that massive cytokines production, leading to cytokine storm syndrome (CSS), played a pivotal role behind COVID-19 pathophysiology in severe and critically ill patients. However, others argued that 
COVID-19 is a disease of different entity and independent from the classic acute respiratory distress syndrome (ARDS) and CSS because IL-6 concentration is much lower in comparison to both diseases. Thus, it is erroneous to say that COVID-19 is identical to ARDS or CSS. Some investigators proposed that COVID-19 is a disease of the blood vessels separated by three phases which ultimately ends with endothelial dysfunction [22-25].

Nevertheless, the number of cytokines in COVID-19 is significantly increased. Those cytokines are IL-2, IL-7, IL-10, tumor necrosis factor (TNF), granulocyte-colony stimulating factor (G-CSF), interferon gamma (IFN- $\gamma$ )-induced protein 10 (IP-10; CXCL10), MCP-1 (CCL2), and MIP-1A (CCL3), which were found to be increased in intensive care unit (ICU) patients compared to non-ICU patients, but not IL-6. Other cytokines that were recently found to contribute in COVID-19 are IL-1 $\beta$, IL-1ra, IL-2R, IL-6, IL-8 (CXCL8), IL-17, IFN- $\gamma$, and granulocyte macrophage colony-stimulating factor (GM-CSF) [26-28]. Three principal pathophysiology, which are T cell immunology, antibody dependent enhancement (ADE) of the virus, and complement activation, are attributed to the resulting aberrant immunological response (GM-CSF). Furthermore, several cytokines such as GM-CSF, IFN- $\gamma$, IL-10, IL-15, IL-8, MCP-1, IL-6, MIP-1 $\beta$, and TNF- $\alpha$ are increased in dengue infection also [17-20]. Health workers and clinicians should keep in mind that COVID-19 and Dengue Fever are hard to distinguish because they share similar clinical features, i.e., clinical manifestations of COVID-19 mimicking Dengue Fever and laboratory findings. The details of these similarities are outside the scope of the present review [18].

Many inflammatory cytokines and chemokines such as IL-6, IL-1 $\beta$, IL-8, CCL8, CXCL9, CXCL16, MCP-1 and IP-10 and immunosuppressive cytokines such as IL-10 are elevated in both infections and associate with clinical disease severity. Although, both COVID-19 and dengue are associated with a cytokine storm and multiorgan involvement, the pathogenesis, disease course and recovery are very much different. Therefore, in order to develop a better insight into both infections and to understand the role of these cytokines in disease pathogenesis and the changes along the course of illness, one should compare the cytokine and chemokine responses in patients with varying severity of acute COVID-19 illness and during the acute/febrile phase and the critical phase of illness [29-35].

However, the levels of IL-6 in those who developed severe COVID-19 were significantly higher than in patients with DHF, and in one of the patients who succumbed to the illness had values of 2,179.7 ng/L 24 hours before death. IL-6 acts on many different cell types resulting in inducing production of other inflammatory cytokines such as IL-8, VEGF, MCP-1 and reduced E-cadherin expression resulting in the increase in the endothelial permeability a contributing to ARDS. Although, increase vascular permeability resulting in plasma leakage and shock is the main pathological feature of DHF, IL-6 appears to contribute less to disease pathogenesis of DHF compared to COVID-19. An impaired type I and type III IFN response in early infection has been shown in COVID-19, especially in older individuals and in those who progress to develop severe disease. It was reported that IFN- $\gamma$ levels were lowest in early illness especially in those who subsequently succumbed to their illness. Although such differences were not observed in those who subsequently developed DHF compared to those with DF, due to the small sample size in this study, it would be important to further characterize these IFN responses in a larger cohort of individuals [29, 30].

The striking observation between those who progressed to develop severe COVID-19 vs DHF was the IL-10 levels. It was shown that those who developed severe COVID-19, were significantly more likely to have IL-10 levels of $>9.1 \mathrm{ng} / \mathrm{L}$, which was seen in $35.8 \%$ of those with severe disease. Interestingly, while only $2 / 15$ patients with COVID-19 had levels exceeding >9.1ng/L, all patients with both DF and DHF had levels far above this value. In fact, the serum IL-10 levels in early illness in those who progressed to develop DHF were 25-fold higher than in those who developed severe COVID-19. Serum IL-10, IL-6 and TNF $\alpha$ levels were shown to negatively correlate with the T cell numbers and IL-10 was shown to associate with T cell exhaustion, suggesting a role in disease pathogenesis [30, 36-38].

Dengue NS1 was shown to induce IL-10 production by monocytes and the persistence of NS1 antigenaemia in patients with acute dengue, correlated with the persistent high levels of IL-10. Collectively, these data suggest that while IL-10 is likely to play a significant role in disease pathogenesis in both COVID-19 and dengue, the contribution to pathogenesis of severe dengue may be greater [39]. Soluble CD40L in serum has been shown to be almost exclusively derived from platelets, which activate CD40 bearing cells to produce cytokines, chemokines and lipid mediators. Increase in soluble CD40L is associated with an increased risk of thrombotic effects and acute lung injury. Thromboembolic events such as deep vein thrombosis and pulmonary embolism have been frequently reported in patients with severe COVID-19 along with ARDS. Therefore, high levels of CD40L especially during the critical phase of the illness could be contributing to the occurrence of these complications [40, 41].

In summary, the changes in several cytokines and chemokines in patients with varying severity of acute 
COVID-19 during different time points in illness should be assessed. Those who develop severe pneumonia in COVID-19 will have high levels of many inflammatory cytokines and chemokines such as IL-10, but low IFN- $\gamma$ levels. Low IFN- $\gamma$ response to the SARS-CoV2 and high levels of an immunosuppressive cytokine such IL-10 in COVID-19 during early phase of illness is likely to result in altered antiviral responses in patients.

\section{Conclusions}

Medical research in the field of 'cytokines in clinical medicine' has been revolutionized with the availability of recombinant cytokines and some of the corresponding monoclonal antibodies. Firstly, the potential therapeutic impacts of cytokines, e.g., IFN- $\gamma$, IL-2, IFN- $\alpha$, G-CSF, GM-CSF, TNF, antibodies to TNF, have been felt mainly in the areas of infectious diseases, cancer, blood disorders, rheumatic and autoimmune diseases. However, potentially dangerous side effects occur with cytokine therapy having implications in clinical medicine. Secondly, it has enormous implications in laboratory medicine also in terms of trained manpower, equipment and costs for commercially available assay kits including efficiency, accuracy and efficacy of the assay kits. Thus, cytokine therapy or clinical trial should be introduced in centres where highly trained and well-informed physicians, nursing staff and laboratory manpower are available. The concern health authorities should make provisions for availability of these specially trained manpower, equipment and logistics in their national health service planning to facilitate the introduction of cytokines in clinical medicine in the third world countries in the very near future. Clinicians should keep in mind that COVID-19 and Dengue Fever are hard to distinguish because they share similar clinical features, i.e., clinical manifestations of COVID-19 mimicking Dengue Fever and laboratory findings. Finally, the cytokine therapy is the area where one can expect the greatest impact of molecular biology and recombinant DNA in clinical medicine in the near future. One can speculate in many ways in which growth factors, colony stimulating factors and other cytokines and their antagonists, receptors and their antagonists will be developed and engineered in the coming years. However, vaccines already developed and being developed in the near future remain the cornerstone for prevention and finally eradication of COVID-19 from this planet.

\section{Acknowledgments}

The authors would like to appreciate efforts given by Mr. Md. Mohiuddin Faisal, Computer Programmer, Impulse Hospital, ImHS\&RCLtd, Dhaka, Bangladesh for helping with the references and computer composing the manuscript.

\section{References}

[1] Balkwill, F. R., Burke, F. (1989). The cytokine networks. Immunology Today, 10: 299-302.

[2] Chapel, H., Haeney, M., Mishbah, S., Snowden, N. (Eds). (2014). Essentials of Clinical Immunology. $6^{\text {th }}$ Edition; UK: Wiley Blackwell Publication.

[3] Giasuddin, A. S. M., Ziu, M. M. (1996). Views for future development of cytokines in health and disease: Implications for clinical medicine. Journal of Islamic Academy of Sciences, 9(3): 67-74.

[4] Callard, R. E., Mathews, D. J., Hibbet. I. (1996). IL-4 and IL-13 receptors; are they one and the same? Immunology Today, 17:108-110.

[5] Burtis, C. A., Bruns, D. E., Sawyer, B. G. (Eds). Tietz Fundamentals of Clinical Chemistry and Molecular Biology, $7^{\text {th }}$ Edition; ST. Louis, Missouri (USA): Elsevier Saunders, 2015.

[6] Romagnani, S. (1991). Human TH1 and TH2 subsets; doubt no more? Immunology Today, 12: 256-257.

[7] Adorini, L., Sinigaglia, F. (1997). Pathogenesis and immunotherapy of autoimmune diseases. Immunology Today, 18: $209-211$.

[8] Symposium on "Future directions of cytokines and immunoglobulin therapy (nine review articles)". (1992). Clinical Immunology and Immunopathology, 62: S1-S65.

[9] Billiau, A., Dijkmans, R. (1990). Interferon gamma: mechanism of action and therapeutic potential. Biochemical Pharmacology, 40: 1433-1441.

[10] Rusell, S. J. (1990). Lymphokine gene therapy for cancer. Immunology Today, 11: 196-201.

[11] Arend, W. P., Dayer, J. M. (1990). Cytokines and cytokine inhibitors or antagonists in rheumatic arthritis. Arthritis and Rheumatism, 33: 305-312.

[12] WHO Director-General's opening remarks at the media briefing on COVID-19: March 2020. Available from: https://www.who.int/dg/speeches/detail/who-director-general-s-opening-remarks-at-the-media-briefing-on-covid-19\%2D\%2D- 
11- march-2020.

[13] Naming the coronavirus disease (COVID-19) and the virus that causes it: 2020 . Available from: https://www.who.int/emergencies/diseases/novel-coronavirus-2019/technical-guidance/naming-the-coronavirus-disease-(covid2019)-and-the- virus-that-causes-it.

[14] Coronavirus Disease 2019 (COVID-19) Situation Report 96: 2020. Available from: https://www.who.int/docs/default-source/ coronaviruse/situation-reports/20200425-sitrep-96-covid19.pdf.

[15] Lorenz, C., Azevedo, T. S., Chiaravalloti-Neto, F. (2020). COVID-19 and dengue fever: A dangerous combination for the health system in Brazil. Travel Medicine and Infectious Disease, 35: 101659. https://doi:10.1016/j.tmaid.2020.101659.

[16] Walls, A. C., Park, Y-J, Tortorici, M. A., Wall, A., McGuire, A. T., Veesler, D. (2020). Structure, function, and antigenicity of the SARS-CoV-2 spike glycoprotein. Cell, 181(2): 281-292.e6.

[17] Astuti, I., Srafil, Y. (2020). Severe acute respiratory syndrome coronavirus 2 (SARS-CoV-2): An overview of viral structure and host response. Diabetes and Metabolic Syndrome, 14(4): 407-412. Doi: 10.1016/j.dsx.2020.04.020. Available from: https://www.ncbi.nlm.nih.gov/pmc/articles/PMC7165108/.

[18] Zhang, J-j, Dong, X, Cao, Y-y, Yuan, Y-d, Yan, Y-B, Yan, Y-Q, et al. (2020). Clinical characteristics of 140 patients infected with SARS-CoV-2 in Wuhan, China. Allergy, 75(7): 1730-1734. https://doi: 10.1111/all.14238.

[19] Gandhi, R. T., Lynch, J. B., del Rio, C. (2020). Mild or moderate Covid-19. New England Journal of Medicine, 383(18): 1757-1766. Available from: http://www.nejm.org/doi/10.1056 / NEJMcp2009249.

[20] Report of the WHO-China Joint Mission on Coronavirus Disease 2019 (COVID-19), 2020. Available from: https://www.who.int/publications-detail/report-of-the-who-china-joint-mission-on-coronavirus-disease-2019-(covid-19).

[21] Hoffmann, M., Kleine-Weber, H., Schroeder, S., Krüger, N., Herrler, T., Erichsen, S., et al. (2020). SARS-CoV-2 cell entry depends on ACE2 and TMPRSS2 and is blocked by a clinically proven protease inhibitor. Cell, 181: 271-280.e8.

[22] Zhang, C., Wu, Z., Li, J.-W., Zhao, H., Wang, G.-Q. (2020). Cytokine release syndrome (CRS) in severe COVID-19: Interleukin-6 receptor (IL-6R) antagonist tocilizumab may be the key to reduce mortality. International Journal of Antimicrobial Agents, 55(5): 105954. https://doi.org/10.1016/j.ijantimicag.2020.105954.

[23] Mehta, P., McAuley, D. F., Brown, M., Sanchez, E., Tattersall, R. S., Manson, J. J. (2020). COVID-19: consider cytokine storm syndromes and immunosuppression. Lancet, 395: 1033-34.

[24] Rahmati, M. (2020). Cytokine-targeted therapy in severely ill COVID-19 patients: options and cautions. Eurasian Journal of Medical Oncology, Available from: https://www.ejmo.org/10.14744/ ejmo.2020.72142.

[25] Leisman, D. E., Deutschman, C. S., Legrand, M. (2020). Facing COVID-19 in the ICU: vascular dysfunction, thrombosis, and dysregulated inflammation. Intensive Care Medicine, 46: 1-4. Available from: http://link.springer.com/10.1007/s00134-02006059-6.

[26] Li, B., Feng, F., Yang, G., Liu, A., Yang, N., Jiang, Q., et al. (2020). Immunoglobulin G/M and cytokines detections in continuous sera from patients with novel coronaviruses (2019-nCoV) infection. Rochester, NY: Social Science Research Network, 2020 Fed/ Report No.: ID 3543609. Available from: https://papers.ssrn.com/ abstract=3543609.

[27] Zhou, Y., Fu, B., Zheng, X., Wang, D., Zhao, C., Qi, Y., et al. (2020). Pathogenic T cells and inflammatory monocytes incite inflammatory storm in severe COVID-19 patients. National Science Review, 7(6): 998-1002. Available from: https://doi.org/10.1093/nsr/nwaa041/5804736.

[28] Zheng, H.-Y., Zhang, M., Yang, C.-X., Zhang, N., Wang, X.-C., Yang, X.-P., et al. (2020). Elevated exhaustion levels and reduced functional diversity of T cells in peripheral blood may predict severe progression in COVID-19 patients. Cellular and Molecular Immunology, 17: 541-543.

[29] Wu, Z., McGoogan, J. M. (2020). Characteristics of and important lessons from the coronavirus disease 2019 (COVID-19) outbreak in China: Summary of a Report of 72314 cases from the Chinese Center for Disease Control and Prevention. Journal of American Medical Association, 323(13): 1239-1242. https://doi:10.1001/jama.2020.2648.

[30] Blanco-Melo, D., Nilsson-Payant, B. E., Liu, W.-C., Uhl, S., Hoagland, D., Møller, R., et al. (2020). Imbalanced host response to SARS-CoV-2 drives development of COVID-19. Cell, 181(5): 1036-1045.e9. https://doi:10.1016/j.cell.2020.04.026 (2020).

[31] Lippi, G., Plebani, M., Henry, B. M. (2020). Thrombocytopenia is associated with severe coronavirus disease 2019 (COVID-19) infections: A meta-analysis. Clinica Chemica Acta, 506: 145-148. https://doi:10.1016/j.cca.2020.03.022.

[32] Guan, W.-j, Ni, Z.-yi, Hu, Y, Liang, W.-h, Ou, C.-q, He, J.-x, et al. (2020). Clinical characteristics of coronavirus disease 2019 in China. New England Journal of Medicine, 382: 1708-1720. https://DOI:10.1056/NEJMoa2002032.

[33] Costela-Ruiz, V. J., Illesces-Montes, R., Puerta-Puerta, J. M., Ruiz, C., Melguizo-Rodriguez. L. (2020). SARS-Cov-2 infection: The role of cytokines in COVID-19 disease. Cytokine and Growth Factor Reviews, 54: 62-75. https://doi:10.1016/cytogfr2020.06.001.

[34] Du, R.-H., Liang, L.-R., Yang, C.-Q., Wang, W., Cao, T.-z, Li. M., et al. (2020). Predictors of mortality for patients with 
COVID-19 pneumonia caused by SARS-CoV-2: a prospective cohort study. European Respiratory Journal, 55(5): 2000524; https://doi:10.1183/13993003.00524-2020.

[35] Wang, K., Zuo, P., Liu, Y., Zhang, M., Zhao, X., Xie, S., et al. (2020). Clinical and laboratory predictors of in-hospital mortality in patients with COVID-19: a cohort study in Wuhan, China. Clinical Infectious Diseases, 71(16): 2079-2088. https://doi.org/:10.1093/cid/ciaa538.

[36] Moore, J. B., June, C. H. (2020). Cytokine release syndrome in severe COVID-19. Science, 368: $473-474$. https://doi:10.1126/science. abb8925.

[37] Diao, B., Wang, C., Tan, Y., Chen, X., Liu, Y., Ning, L., et al. (2020). Reduction and functional exhaustion of T cells in patients with coronavirus disease 2019 (COVID-19). Frontiers in Immunology, 11: 827. https://doi:10.3389/fimmu.2020.00827.

[38] Henrina, J., Putra, I. C. S., Lawrensea, S., Handoyono, Q. F., Cahyadi, A. (2020). Coronavirus disease of 2019: A mimicker of dengue infection? SN Comprehensive Clinical Medicine, 2: 1109-1119. Available from: https://doi.org/10.1007/s42399-020-00364-3.

[39] Adikari, T. N., Gomes, L., Wickramasinghe, N., Salimi, M., Wijesiriwardana, N., Kamaladasa, A., et al. (2016). Dengue NS1 antigen contributes to disease severity by inducing interleukin (IL)-10 by monocytes. Clinical and Experimental Immunology, 184(1): 90-100. https://doi:10.1111/cei.12747.

[40] Blumberg, N., Spinelli, S. L., Francis, C. W., Taubman, M. B., Phipps, R. P. (2009). The platelet as an immune cell-CD40 ligand and transfusion immunomodulation. Immunologic Research, 45(2-3): 251-260. https://doi:10.1007/s12026-009-8106-9.

[41] Wichmann, D., Sperhake, J.-P., Lutgehetmann, M., Steurer, S., Edler, C., Heinemann, A., et al. (2020). Autopsy findings and venous thromboembolism in patients with COVID-19. Annals of Internal Medicine, M20-2003. https://doi:10.7326/M20-2003. 